\title{
KINETICS OF ZOLPIDEM AND ITS METABOLITE AFTER SINGLE DOSE ORAL ADMINISTRATION
}

\author{
ANA-MARIA GHELDIUa, ALEXANDRA CSAVDARI ${ }^{\mathrm{b}}$, MARCELA ACHIM ${ }^{\mathrm{a}}$, \\ LAURIAN VLASE ${ }^{a^{*}}$, IOAN TOMUȚA ${ }^{a}$, DANA MARIA MUNTEAN ${ }^{a}$
}

\begin{abstract}
The present study aimed to describe the basic pharmacokinetics of zolpidem and its metabolite zolpidem phenyl-4-carboxylic acid after a single oral dose of $5 \mathrm{mg}$ zolpidem. Six competing kinetic models were created in order to analyze the experimental data obtained from the 20 healthy volunteers enrolled in a clinical study. Based on rational model discrimination criteria (Akaike index value), the best one was chosen and further used for a better understanding of the kinetics of zolpidem and its metabolite in the body after administration. The kinetic model considers that zolpidem absorption process follows a first-order kinetics and during this stage it is partially metabolized (pre-systemic metabolism) to its main metabolite. The kinetics of both zolpidem and its metabolite is characterized by bicompartmental distribution and first order kinetics of both elimination and systemic metabolism.
\end{abstract}

Keywords: zolpidem, zolpidem phenyl-4-carboxylic acid, compartmental pharmacokinetic analysis

\section{INTRODUCTION}

Zolpidem is an imidazopyridine which acts at the benzodiazepine $\omega 1$-receptor subtype [1,2] exhibiting hypnotic-sedative action exclusively due to agonist binding on the $\alpha_{1}$ - gamma-aminobutyric acid type $A\left(G_{A B A}\right)$

\footnotetext{
a University of Medicine and Pharmacy "Iuliu Hatieganu", Faculty of Pharmacy, Department of Pharmaceutical Technology and Biopharmaceutics, 8 Victor Babes str., RO-400012, Cluj-Napoca, Romania

${ }^{b}$ Babeş-Bolyai University, Faculty of Chemistry and Chemical Engineering, 11 Arany Janos str., RO-400028, Cluj-Napoca, Romania

*Corresponding author: laurian.vlase@yahoo.com
} 
receptors. Zolpidem is recommended for the reduction of sleep onset time, increase total duration of sleep and sleep efficiency, given in doses of 5 up to $7.5 \mathrm{mg}[1,6]$ for the short-term treatment of insomnia [7].

Zolpidem displays rapid absorption after oral administration and has an absolute bioavailability of about $70 \%[5,8]$. Is characterized by linear kinetics in the $5-20 \mathrm{mg}$ dose range [5], is highly bound to plasma proteins (around 92\%) [6] and it is subjected to extensive hepatic metabolism $[7,8]$. Zolpidem is a substrate to several distinct CYP 450 isoenzymes, among which the major metabolism pathway occurs through CYP3A4 $(61 \%)[9,10,11]$. It is converted to three pharmacologically inactive metabolites in the liver via oxidation and hydroxylation, of which the 4-carboxy-derivative is the predominant one (zolpidem phenyl-4-carboxylic acid - Z4CA), representing 72 up to $86 \%$ of the administered dose $[12,13,14]$. The time to reach the maximum plasma concentration is around $0.5-3 \mathrm{hr}$ and the half-life time of zolpidem is about $2-3$ hours $[3,4]$.

Pharmacokinetics, by the quantitative study of the processes that take place depending on time, offers a better understanding of the relationship between the given/administered dose and the pharmacological effect [15]. The compartmental modeling approach of pharmacokinetics consists in describing the processes that the administered drug is subjected to in the body, depicted as an entity divided into distinct compartments with different properties and specific affinities for the drug or drug metabolites [16]. The drug leaves the site of administration by the process of absorption in order to reach the central compartment from which is it further exchanged both-ways with the peripheral compartments (distribution process) and it is later irreversibly eliminated from the body (by metabolism and/or excretion). All the kinetic processes that the drug undergoes in the body can be characterized by transfer rate constants, which in linear kinetics (1st order kinetics) are assumed to be directly proportional to the mass of transfer available drug $[1,8]$. By performing compartmental and non-compartmental analysis, the corresponding pharmacokinetic parameters of the drug can be obtained, and they can be further used in drug formulation development [17], bioequivalence assays or in therapeutic drug monitoring for patient-specific dose adjustment [18].

The aim of this study was to create and to use a pharmacokinetic model that can accurately describe the kinetic processes involved in absorption, distribution, metabolism and elimination of zolpidem and zolpidem phenyl-4-carboxylic acid (Z4CA) after oral administration of a single dose of zolpidem in healthy volunteers, by comparing predicted values with actual experimental data. 


\section{RESULTS AND DISCUSSION}

A number of six distinct mathematical models were created with the purpose of assessing the pharmacokinetics of zolpidem and its main metabolite, Z4CA. The characteristics of each individual kinetic model are summarized in Table 1.

Table 1. Pharmacokinetic models of zolpidem and its metabolite used in compartmental analysis

\begin{tabular}{|c|c|c|c|c|}
\hline Model & $\begin{array}{c}\text { Pre-systemic } \\
\text { metabolism } \\
\text { kinetics }\end{array}$ & $\begin{array}{c}\text { Systemic } \\
\text { metabolism } \\
\text { kinetics }\end{array}$ & $\begin{array}{c}\text { Zolpidem, } \\
\text { number of } \\
\text { compartments }\end{array}$ & $\begin{array}{c}\text { Zolpidem phenyl-4- } \\
\text { carboxylic acid, number } \\
\text { of compartments }\end{array}$ \\
\hline M1 & No process & $1^{\text {st }}$ order & 1 & 1 \\
\hline M2 & No process & $1^{\text {st }}$ order & 2 & 1 \\
\hline M3 & No process & $1^{\text {st }}$ order & 2 & 2 \\
\hline M4 & $1^{\text {st }}$ order & $1^{\text {st }}$ order & 1 & 1 \\
\hline M5 & $1^{\text {st }}$ order & $1^{\text {st }}$ order & 2 & 1 \\
\hline M6 & $1^{\text {st }}$ order & $1^{\text {st }}$ order & 2 & 2 \\
\hline
\end{tabular}

The differences between the evaluated mathematical models consisted in suppositions about the existence of pre-systemic metabolism of zolpidem and about the number of compartments for both zolpidem and Z4CA. For instance, the first pharmacokinetic model (M1) assumes no presystemic metabolism of zolpidem and monocompartmental distribution for both zolpidem and its metabolite. The model M6 employs existence of presystemic metabolism and bicompartmental distribution for both zolpidem and Z4CA. For each tested model, the process of compounds elimination along with the systemic metabolism of zolpidem to zolpidem phenyl-4-carboxylic acid were regarded as 1 st order kinetic processes.

The schematic representation of the kinetic processes from model M6 are shown in Figure 1. 


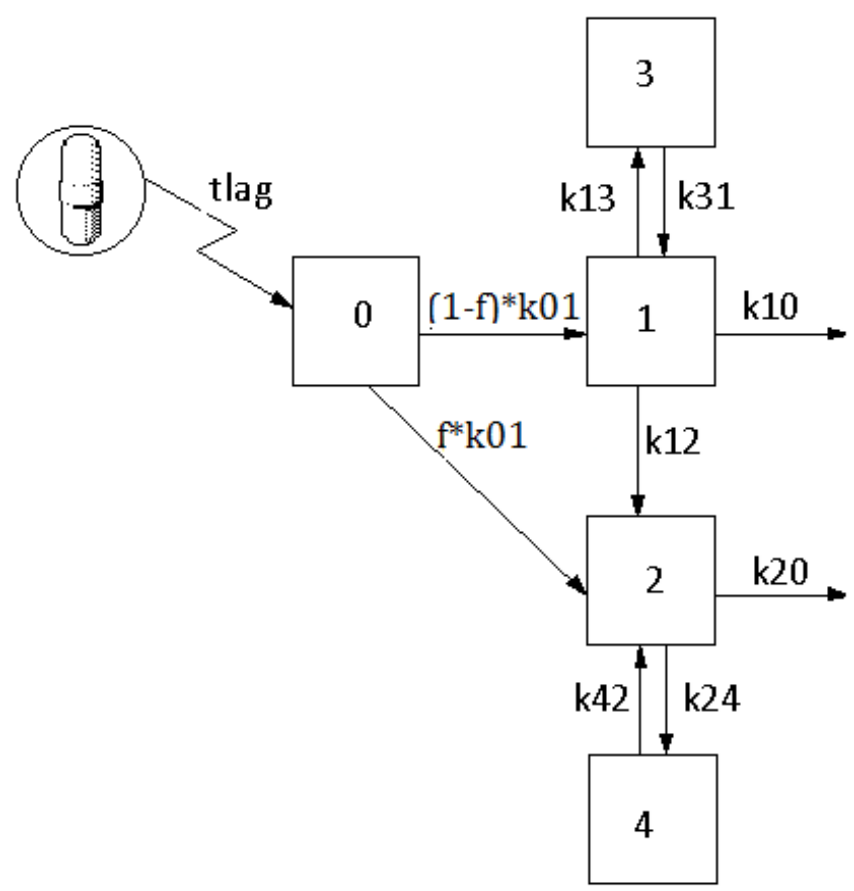

Figure 1. Schematic representation of kinetic processes from model M6, where " 0 " is absorption compartment of zolpidem; " 1 " and "2" are central compartments of zolpidem and zolpidem phenyl-4-carboxylic acid; "3" and "4" are their corresponding peripheral distribution compartments; tag is the latency time for absorption; k01 is the absorption rate constant of zolpidem, $\mathrm{f}$ is the fraction of zolpidem converted into metabolite during absorption (pre-systemic metabolism); k13, k31, k24, k42 are the distribution rate constants; $\mathrm{k} 12$ is the systemic metabolization rate constant of zolpidem to metabolite; k10 and k20 are the elimination rate constants for zolpidem (non-metabolic) and zolpidem phenyl-4-carboxylic acid. The kinetic processes are represented by straight arrows.

For each analyzed kinetic model, the corresponding mathematical differential equations were written and run by using Phoenix 6.1 software package (Certara, SUA). The equations of model M6 are illustrated in Figure 2. 


$$
M 6\left\{\begin{array}{l}
\frac{\partial Q Z_{a b s}}{\partial t}=-k 01 * Q Z_{a b s} \\
\frac{\partial Q Z_{C}}{\partial t}=(1-f) * k 01 * Q Z_{a b s}-k 10 * Q Z_{C}-k 13 * Q Z_{C}+k 31 * Q Z_{P}-k 12 * Q Z_{C} \\
\frac{\partial Q Z_{P}}{\partial t}=k 13 * Q Z_{C}-k 31 * Q Z_{P} \\
\frac{\partial Q M_{C}}{\partial t}=f * k 01 * Q Z_{a b s}+k 12 * Q Z_{C}-k 20 * Q M_{C}-k 24 * Q M_{C}+k 42 * Q M_{P} \\
\frac{\partial Q M_{P}}{\partial t}=k 24 * Q M_{C}-k 42 * Q M_{P} \\
\text { ConcZc }=\frac{Q Z_{C}}{V_{-} F} \\
\text { ConcMc }=\frac{Q M c}{V_{-} F}
\end{array}\right.
$$

Figure 2. The mathematical equations of the kinetic model $M 6$, where $Q_{c}$ and $Q Z_{p}$ are the amount of zolpidem in central and peripheral compartment respectively; QMc and QMp are the quantities of metabolite in central and peripheral compartments; ConcZ and ConcM are the plasma concentrations of zolpidem and zolpidem phenyl4-carboxylic acid, V_F is the apparent volume of distribution of the central compartment. All the other parameters used were previously presented

The mean plasma concentrations of zolpidem and zolpidem phenyl4-carboxylic acid were evaluated using the six kinetic models previously described, after their implementation in Phoenix software. It was used the same settings of the software minimisation engine for all models analysis: weighting scheme 1/y (1/observed concentration), minimisation method: Gauss-Newton (Levenberg and Hartley variant), convergence criterion: 0.0001 .

The Akaike index (automatically calculated and provided by the analysis software) was used for model discrimination $[22,23]$. The model that proved a better fitting to the experimental data was characterised by a smaller Akaike index. The Akaike values for the six analysed models are presented in Figure 3.

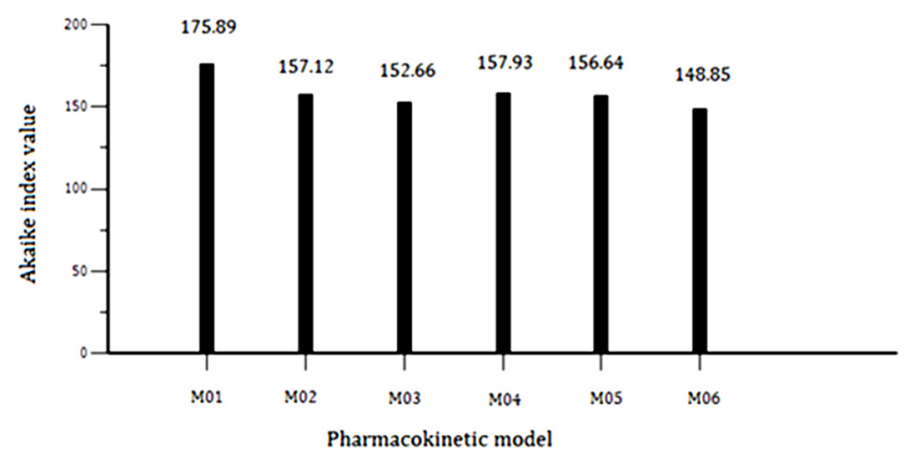

Figure 3. Akaike index values for mathematical models used for characterisation of zolpidem and zolpidem phenyl-4-carboxylic acid pharmacokinetics 
By visually inspecting the Akaike values presented in Figure 3, it can easily be observed that model M6 fits the experimental data better than its concurrent models, displaying the smallest Akaike value, therefore it was elected as representative for describing the kinetics of zolpidem and Z4CA after oral administration of a single dose of zolpidem.

Figure 4 presents a typical fitting of a subject dataset to representative model M6 in comparison with M1. Zolpidem and Z4CA plasma concentrations displayed a better correlation between the experimental (observed) and the fitted (predicted) values in case of model M6 than in case of model M1.
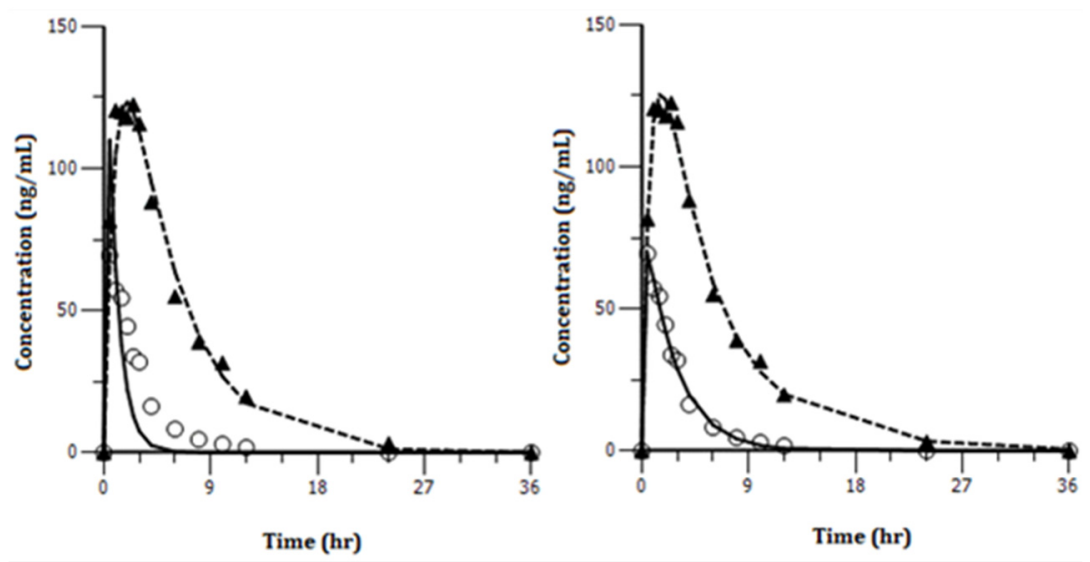

Figure 4. Typical fitting of model M1 (left) and model M6 (right) to a subject dataset ( $\circ$ and $\boldsymbol{\Lambda}$ denote the experimental determined concentrations of zolpidem and its metabolite, respectively; the continues and dotted lines are the fitted/computed concentrations predicted by the kinetic model)

According to kinetic model M6, the pharmacokinetics of zolpidem is characterised by a first order absorption kinetics with pre-systemic metabolism to zolpidem phenyl-4-carboxylic acid. Once inside the body, both zolpidem and Z4CA are characterised by bicompartmental distribution. After absorption, zolpidem is subject to systemic metabolism leading to the formation of the main metabolite, zolpidem phenyl-4-carboxylic acid, following a first-order kinetic process. Both compounds are further eliminated from the body by first order kinetic processes. By using this representative pharmacokinetic model for zolpidem and Z4CA, their characteristic pharmacokinetic parameters were calculated (Table 2). 
Table 2. The kinetic parameters of zolpidem and zolpidem phenyl-4-carboxylic acid calculated with model M6

\begin{tabular}{|c|r|r|r|r|r|}
\hline Variable & \multicolumn{1}{|c|}{ Mean } & \multicolumn{1}{c|}{ SD } & \multicolumn{1}{c|}{ \% CV } & \multicolumn{1}{c|}{ Median } & Geometric mean \\
\hline$T_{\text {lag }}(\mathrm{hr})$ & 0.281 & 0.251 & 89.2 & 0.327 & 0.0248 \\
\hline $\mathrm{k} 01\left(\mathrm{hr}^{-1}\right)$ & 0.644 & 0.603 & 93.7 & 0.367 & 0.47 \\
\hline $\mathrm{f}$ & 0.311 & 0.177 & 57 & 0.251 & 0.226 \\
\hline $\mathrm{k} 10\left(\mathrm{hr}^{-1}\right)$ & 0.135 & 0.164 & 121 & 0.0733 & 0.026 \\
\hline $\mathrm{k} 12\left(\mathrm{hr}^{-1}\right)$ & 3.54 & 2.98 & 84.3 & 2.82 & 1.37 \\
\hline $\mathrm{k} 13\left(\mathrm{hr}^{-1}\right)$ & 4.66 & 3.92 & 84.2 & 3.48 & 2.44 \\
\hline $\mathrm{k} 31\left(\mathrm{hr}^{-1}\right)$ & 2.21 & 3.09 & 140 & 0.255 & 0.358 \\
\hline $\mathrm{k} 20\left(\mathrm{hr}^{-1}\right)$ & 2.37 & 2.37 & 100 & 2.04 & 1.5 \\
\hline $\mathrm{k} 24\left(\mathrm{hr}^{-1}\right)$ & 2.08 & 2.36 & 113 & 1.12 & 0.451 \\
\hline $\mathrm{k} 42\left(\mathrm{hr}^{-1}\right)$ & 1.69 & 2.08 & 123 & 0.826 & 0.608 \\
\hline V_F $(\mathrm{L})$ & 5660 & 4330 & 76.5 & 3690 & 4030 \\
\hline
\end{tabular}

A considerable variability of calculated kinetic parameters of zolpidem and its metabolite can be observed between the 20 subjects enrolled in the study (Table 2). However, this is currently observed in clinical studies, involving human subjects participation, due to natural biological and physiological differences between subjects (inter-subject variability) [24,25].

The absorption of zolpidem is delayed for about $0.28 \pm 0.25$ hours after oral administration, the time needed for the biopharmaceutical processes to take place (disintegration, release, dissolution of the drug molecules in the liquid interior body medium) and for the drug molecules to reach into duodenum. The absorption rate constant is $0.644 \pm 0.603 \mathrm{hr}-1$, which means an absorption half-life time of about $1.076 \mathrm{hr}$. During the stage of absorption, about $30 \%$ of the bioavailable amount of zolpidem is converted to inactive metabolite which appears in plasma, meaning that the oral bioavailability of zolpidem (pharmacologically active molecule) is approximately $70 \%$.

The apparent volume of distribution for central compartment of both zolpidem and metabolite is about $5660 \pm 4330 \mathrm{~L}$, this high value being expected as both compounds are lipophilic and highly bounded on tissue proteins (approximately 92\% protein binding). The kinetic model M6 considers two possible elimination pathways for zolpidem: by systemic metabolism to zolpidem phenyl-4-carboxylic acid (characterised by a rate constant k12) and by other processes, primarily by renal excretion (characterised by an overall rate constant k10). As it can be observed from Table 2, the value of $k 12$ $(3.54 \pm 2.98 \mathrm{hr}-1)$ is much higher than $\mathrm{k} 10(0.135 \pm 0.164 \mathrm{hr}-1)$. This means that the majority of zolpidem $(99.6 \%)$ is eliminated from the body by metabolism to Z4CA, the rest being eliminated by metabolization to other metabolites or by direct renal excretion. Both zolpidem and its metabolite are distributed between central and peripheral compartments, the latter exhibiting a higher affinity for each compound (k13>k31 and k24>k42). 
The observed plasma concentrations of zolpidem phenyl-4-carboxylic acid are due to both pre-systemic and systemic biotransformation of zolpidem. The metabolite is eliminated following a first-order kinetic process, characterised by a rate constant of $2.37 \pm 2.37 \mathrm{hr}-1$.

\section{CONCLUSIONS}

Six different mathematical models were tested in order to describe the kinetics of zolpidem and its metabolite Z4CA after oral administration of a single oral dose of $5 \mathrm{mg}$ zolpidem. These models involved differences regarding the pre-systemic metabolism of zolpidem to its metabolite and the mono- or bicompartmental distribution of the compounds in the body.

After experimental data analysis, the representative model for the pharmacokinetics of zolpidem and its metabolite was found and described. The model considers that zolpidem is absorbed following a first-order kinetic process and is partially converted during absorption to its main metabolite, Z4CA. The kinetics of zolpidem is characterized by bicompartmental distribution and first order kinetic elimination processes $(99.6 \%$ by biotransformation to metabolite, the rest by other paths). The metabolite zolpidem phenyl-4-carboxylic acid displays also a bicompartmental distribution and a first order elimination kinetics.

The knowledge of drug kinetics in the body through compartmental modeling is the starting point for other important analysis such as pharmacokinetic population modelling, prediction of drug plasma levels at other doses or when multiple doses are administered or further mathematical correlations between drug kinetics and pharmacological effect intensity. It also allows a better calculation of dosage regimen of a drug in a particular situation, considering the influence of altered physiology or disease state on drug absorption, distribution, metabolism and elimination as well as giving a better understanding of drug interactions, if the case.

\section{EXPERIMENTAL SECTION}

Subjects: In this study were enrolled 20 healthy volunteers and all gave their written informed consent prior to study inclusion. The study was conducted according to the principles of Declaration of Helsinki (1964) and its amendments (Tokyo 1975, Venice 1983, Hong Kong 1989). The clinical protocol was reviewed and approved by the Ethics Committee of the University of Medicine and Pharmacy "Iuliu Hatieganu", Cluj-Napoca, Romania. 
Study protocol: After an overnight fast, the volunteers received a single $5 \mathrm{mg}$ zolpidem dose at 8:00 a.m. with $150 \mathrm{ml}$ of water. The pharmaceutical product used was Stilnox (10 mg film-coated tablets, Sanofi-Aventis - Romania). Venous blood samples $(5 \mathrm{ml})$ were taken according to the following time schedule: before drug administration $(0 \mathrm{~h})$, and at $0.5,1,1.5,2,2.5,3,4,6,8$, $10,12,24,36$ and 48 hours after drug administration. Within 10 minutes of collection, blood samples were centrifuged at $5000 \mathrm{rpm}$ for 10 minutes and plasma samples were frozen stored at $-20^{\circ} \mathrm{C}$ until further analysis.

Drug analysis from plasma: Zolpidem and Z4CA plasma concentrations were determined using a validated high-throughput liquid chromatography tandem mass spectrometry method. The HPLC system was an Agilent 1100 series (binary pump, autosampler, thermostat) (Agilent Technologies, USA) and was coupled with a Brucker lon Trap SL (Brucker Daltonic s GmbH, Germany). A Zorbax SB-C18 chromatographic column (100 mm x $3.0 \mathrm{~mm}$ i.d., $3.5 \mu \mathrm{m})$ (Agilent Technologies) was used.

The mobile phase was a mixture of $2 \mathrm{mM}$ ammonium formate solution and acetonitrile, elution in gradient: $11 \%$ acetonitrile at start, $41 \%$ at 2 minutes. The flow rate was $1 \mathrm{ml} / \mathrm{min}$ and the thermostat temperature was set at $48^{\circ} \mathrm{C}$. The mass spectrometry detection was in multiple reaction monitoring mode, positive ions, using an electrospray ionization source. The ion transitions monitored were for zolpidem were $\mathrm{m} / \mathrm{z}(235.5 ; 263.2)$ from 308 and for its metabolite $\mathrm{m} / \mathrm{z}$ (265.1; 266.1; 293.1) from 338, respectively $[19,20,21]$. The calibration curves for both zolpidem and its metabolite were linear between $2-400 \mathrm{ng} / \mathrm{ml}$.

Pharmacokinetic analysis: The compartmental pharmacokinetic analysis was performed in order to analyze the plasma versus time levels of zolpidem and its metabolite for each individual dataset obtained from volunteers (20 datasets).

Six distinct mathematical models were created in order to assess the pharmacokinetics of zolpidem and its metabolite (see Table 1).

\section{REFERENCES}

1. Langtry H.D., Benfield P., Drugs, 1990, 40(2), 291.

2. Hoehns J.D., Perry P.J., Clinical Pharmacy, 1993, 12(11), 814.

3. Vlase L., Popa A., Neag M. Muntean D., Leucuta S.E., European Journal of Drug Metabolism and Pharmacokinetics, 2011, 35(3), 83.

4. Vlase L., Popa A., Neag M., Muntean D., Baldea I., Leucuta S.E., Journal of Clinical Pharmacology, 2011, 51(8), 1233.

5. Swainston Harrison T, Keating GM., CNS Drugs, 2005, 19(1), 65.

6. Holm K.J., Goa K.L., Drugs, 2000, 59(4), 865. 
7. Darcourt G, Pringuey D, Sallière D, Lavoisy J., Journal of Psychopharmacology, 1999, 13(1), 81.

8. Drover DR., Clinical Pharmacokinetics. 2004, 43(4), 227.

9. Barkin R.L., American Journal of Therapeutics, 2007, 14(3), 299.

10. Greenblatt DJ, Roth T., Expert Opinion on Pharmacotherapy, 2012, 13(6), 879.

11. Von Moltke LL, Greenblatt DJ, Granda BW, Su XD, Grassi JM, Venkatakrishnan K, et al., British Journal of Clinical Pharmacology, 1999, 48, 89.

12. Krylova E a., Kataev SS, Khomov Y, Journal of Analytical Chemistry, 2013, 68(8), 722.

13. Zhou SF, Zhou ZW, Yang LP, Cai JP, Current Medicinal Chemistry, 2009, 16(27), 3480.

14. Pichard L, Gillet G, Bonfils C, Domergue J, Thenot JP, Maurel P, Drug Metabolism and Disposition, 1995, 23(11), 1253.

15. Benet L.Z., Zia-Amirhosseini P., Toxicologic Pathology, 1995, 23(2), 115.

16. Himmelstein K.J., Lutz R.J., Journal of Pharmacokinetics and Biopharmaceutics, 1979, 7(2), 127.

17. Peck C.C., Barr W.H., Benet L.Z., Collins J., Desjardins R.E., Furst D.E., et al, Journal of Pharmaceutical Sciences, 1992, 81(6), 605.

18. Mangoni A.A., Jackson S.H.D., British Journal of Clinical Pharmacology, 2003, 57(1), 6.

19. Gheldiu A.M., Popa A., Neag M., Muntean D., Bocsan C., Buzoianu A., Vlase L., Achim M., Todor I., Briciu C., Farmacia, 2015, 63(3), 453.

20. Vlase L., Popa A., Neag M., Muntean D., Baldea I., Leucuta S.E., Journal of Clinical Pharmacology, 2011, 51, 1233.

21. Vlase L., Popa A., Neag M., Muntean D., Leucuta S.E., European Journal of Drug Metabolism and Pharmacokinetics, 2011, 35, 83.

22. Akaike H., IEEE Transactions on Automatic Control, 1974, 19, 716.

23. Ben-Akiva M, Swait J., Transportation Science, 1986, 20(2), 133.

24. Lin J. H., Current Drug Metabolism, 2007, 8(2), 109.

25. Leucuta S. E., Vlase L., Current Clinical Pharmacology, 2006, 1(1), 5. 